\title{
BMP4 is increased in the aortas of diabetic ApoE knockout mice and enhances uptake of oxidized low density lipoprotein into peritoneal macrophages
}

Mitsuhisa Koga, Atsushi Yamauchi, Yuki Kanaoka, Ryusuke Jige, Anna Tsukamoto, Nao Teshima, Tsuyoshi Nishioku and Yasufumi Kataoka*

\begin{abstract}
Background: BMP4, a member of the transforming growth factor-beta superfamily, is upregulated in the aortas of diabetic $\mathrm{db} / \mathrm{db}$ mice. However, little is known about its role in diabetic atherosclerosis. Therefore, we examined the roles of BMP4 in the formation of diabetic atherosclerosis in apolipoprotein E knockout (ApoE KO) mice and in the uptake of oxidized low density lipoprotein (oxLDL) in peritoneal macrophages of wild-type mice.

Methods: To induce diabetes, ApoE KO mice were intraperitoneally injected with streptozotocin. Diabetic and non-diabetic ApoE KO mice were then fed a high-fat diet for 4 weeks. Next, to investigate a role of BMP4 in the peritoneal macrophages, we examined the uptake of oxLDL in BMP4-treated macrophages.

Results: Diabetic ApoE KO mice showed accelerated progression of aortic plaques accompanied by increased luminal plaque area. Western blot analysis showed that BMP4 expression in the whole aorta was greatly increased in diabetic ApoE KO mice, than non-diabetic mice. Western blot analysis showed that the BMP4/SMAD1/5/8 signaling pathway was strongly activated in the aorta from diabetic ApoE KO mice, compared with control ApoE KO mice. Double immunofluorescence staining showed that BMP4 was expressed in MOMA2-labeled macrophage in the aortic lesions of ApoE KO mice. BMP4 significantly increased the uptake of oxLDL into peritoneal macrophages in vitro.

Conclusion: We show that in the aorta of diabetic ApoE KO mice, BMP4 is increased and activates SMAD1/5/8. Our in vitro findings indicate that BMP4 enhances oxLDL uptake in mouse peritoneal macrophages, suggesting BMP4 may be involved in aortic plaque formation in diabetic ApoE KO mice. Targeting BMP4 may offer a new strategy for inhibition of plaque progression and stabilization of atherosclerotic lesions.
\end{abstract}

Keywords: BMP4, Atherosclerosis, Diabetes, Macrophage, Oxidized low density lipoproteins

\section{Introduction}

Diabetes accelerates the progression of atherosclerosis, and induces vascular complications that are often life-threatening and disabling [1]. These complications represent a major clinical problem. There is increasing evidence that atherosclerosis is a chronic inflammatory disease in which inflammatory cells, including macrophages, monocytes, and T-lymphocytes, are recruited to and are

\footnotetext{
* Correspondence: ykataoka@fukuoka-u.ac.jp

Department of Pharmaceutical Care and Health Sciences, Faculty of Pharmaceutical Sciences, Fukuoka University, 8-19-1 Nanakuma, Jonan-ku, Fukuoka 814-0180, Japan
}

activated in the atherosclerotic plaque by various cytokines and chemokines. Previous studies have revealed that oxidized low-density lipoprotein (oxLDL) is a causal factor for cardiovascular diseases [2-4]. An accumulation of oxLDL in foam cells derived from macrophages in atherosclerotic plaques causes plaque instability, before rupture $[5,6]$. These macrophages play key roles in all stages of atherosclerosis.

Bone morphogenetic proteins (BMPs) are bone-inducing morphogens and belong to the members of transforming growth factor- $\beta$ superfamily $[7,8]$. BMPs also modulate cellular differentiation, proliferation, lineage determination, 


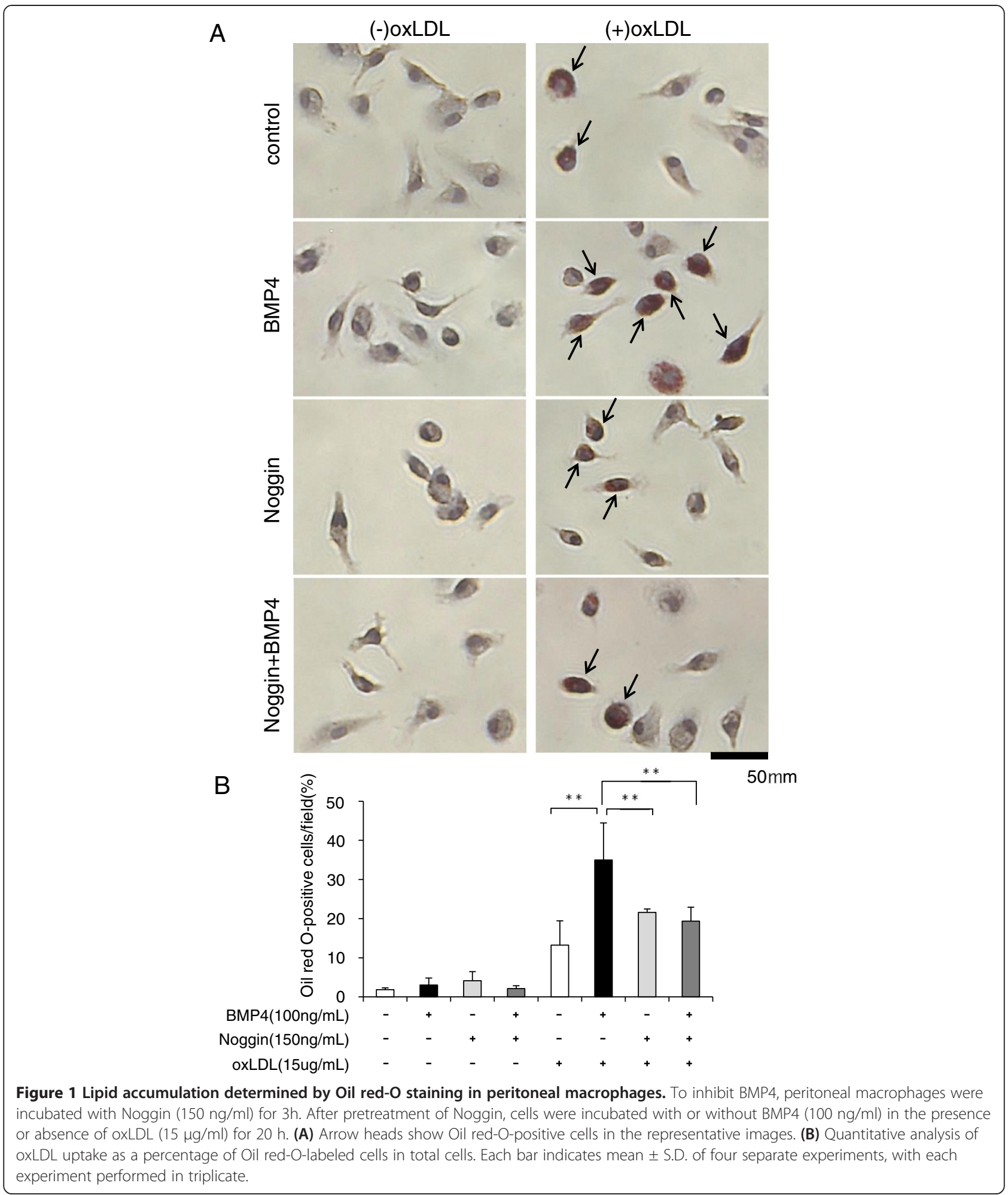

motility, and death [9-13]. Although the functions of BMPs in embryogenesis have been extensively studied, their roles after birth remain unclear [11].

In particular, BMP4 is expressed in calcified atherosclerotic plaques and aortic valve diseases [14], and these vascular BMPs contribute to the development of cardiovascular diseases. BMP4 is upregulated in $d b / d b$ mice, an animal model of diabetes [15,16]. BMP4 was also reported to mediate monocyte adhesion, which is enhanced in atherosclerosis $[17,18]$, restenosis [19], and diabetes [15]. 
Table 1 Non-fasting glucose, lipid, and lipoprotein levels in control and diabetic mice

\begin{tabular}{lcc}
\hline & cont & STZ \\
\hline Body Weight $(\mathrm{g})$ & $23.6+/-2.8$ & $21.2+/-3.5^{*}$ \\
Glucose $(\mathrm{mg} / \mathrm{dl})$ & $102+/-12$ & $454+/-123^{* *}$ \\
Total Cholesterol (mg/dl) & $1815+/-302$ & $2529+/-513^{* *}$ \\
Triglycerides $(\mathrm{mg} / \mathrm{dl})$ & $499+/-74$ & $548+/-164$ \\
\hline
\end{tabular}

Data are expressed as mean +/- S.D. obtained from 16 diabetic ApoE mice and 18 control mice. ${ }^{*} \mathrm{P}<0.05$ and ${ }^{* *} \mathrm{P}<0.01$ vs control mice.

Chronic BMP4 infusion causes endothelial dysfunction in a vascular NADPH oxidase-dependent manner in mice. Therefore, BMP4 is likely to be involved in the induction of hypertension [20]. Other BMPs, which have antagonistic properties, are coexpressed with BMP4 in mouse aortas and in human coronary arteries, suggesting that BMPs, including BMP4, are involved in the formation of atherosclerosis [21]. Taken together, these findings support the notion that BMPs play an important role in the pathophysiology of cardiovascular diseases and that they are key mediators of atherosclerosis. However, little is known about the role of BMP4 in macrophages in atherosclerotic plaques. Therefore, we examined the expression levels of BMP4 in atherosclerotic plaques of streptozotocin (STZ)induced diabetic ApoE KO mice and the role of BMP4 in oxLDL uptake into macrophages in atherosclerotic lesions.

\section{Methods}

\section{Animals}

C57BL/6J ApoE KO mice were purchased from Jackson Laboratory (Bar Harbor, ME, USA) and were housed under standard conditions, including humidity, room temperature, and dark-light cycles. Mice were given free access to food and water throughout the study. The study protocol was approved by the Laboratory Animal Care and Use Committee of Fukuoka University. ApoE $\mathrm{KO}$ mice (6 weeks old) were intraperitoneally injected with $55 \mathrm{mg} \mathrm{kg}^{-1}$ day $^{-1} \mathrm{STZ}$ or vehicle (citrate buffer, control) over 5 consecutive days [22,23]. Blood glucose levels were measured 2 weeks after STZ administration to assess the induction of diabetes; only diabetic mice (defined as non-fasting glucose $>250 \mathrm{mg} / \mathrm{dL}$ ) were used in this study. Both groups of ApoE KO mice were fed a high-fat diet $(1.25 \%$ cholesterol, $15 \%$ cacao butter, and $0.5 \%$ sodium cholate, F2HFD1, Oriental Yeast CO, Tokyo, Japan) for 4 weeks, starting from 8 weeks old. At 12 weeks old, the animals were killed, and the aortas removed for comparisons between STZ-induced diabetes and control mice.

\section{En-face plaque area}

To quantify the extent of atherosclerotic lesions, immediately after the mice were killed, the whole length of the aorta ( $\mathrm{n}=9$ mice in each group) was excised for quantification of the en face plaque area, as previously described [24,25]. Briefly, after carefully removing adventitial tissue, the aortic arch and the thoracic to abdominal aorta were opened longitudinally, pinned on a black wax surface, and stained with Oil red $\mathrm{O}$ (Sigma, St. Louis, MO, USA). En face images were obtained by a stereomicroscope and analyzed using a public domain software Image J (NIH Image, Bethesda, MD, USA) ( $\mathrm{n}=9$ mice/group). The percentage of the luminal surface area stained by Oil red-O was determined [24,25].

\section{Histology}

After the mouse was sacrificed and perfused with ice-cold phosphate-buffered saline (PBS), the heart and the ascending aorta were removed en bloc and snap-freezed in O.C.T. compound (Sakura FineTech, Tokyo, Japan) for histological and immunohistochemical analyses. Serial cryostat sections (6 $\mu \mathrm{m}$ thick) of the aortic root were prepared as previously described [24,25]. Briefly, atherosclerotic plaques were examined in five independent sets of sections taken $60 \mu \mathrm{m}$ apart. Oil red $\mathrm{O}$ staining was performed to identify the lipid-rich core. The Oil red O-stained areas, as a marker of lipid accumulation, were analyzed using Image J software. In each mouse, the mean for five independent sections was used for the analysis.

\section{Double immunofluorescence staining}

For staining the frozen sections, fresh mouse aortas were excised from ApoE KO mice, placed in Tissue-Tek O.C.T. compound, snap-freezed in lipid nitrogen, and stored at $-80^{\circ} \mathrm{C}$ until use. After removing the O.C.T. compound and blocking, the samples were incubated with antibodies against BMP4 (1:100; Santa Cruz Biotechnology Inc., Santa Cruz, CA, USA) and MOMA2 (1:500; BMA Biomedicals, Augst, Switzerland) overnight at $4^{\circ} \mathrm{C}$. For double-immunofluorescence staining, the samples were incubated with FITC (Nacalai Tesque, Kyoto, Japan) and AlexaFluor 594-conjugated secondary antibodies (Molecular Probes, Eugene, OR, USA), respectively, for $1 \mathrm{~h}$ at room temperature. Nuclei were counterstained with DAPI (Vector Laboratories, Burlingame, CA, USA). MOMA2-stained areas, as a marker of macrophage accumulation, were analyzed using Image J software.

\section{Western blotting}

The aorta was immediately snap-freezed in liquid nitrogen. Aortic proteins were isolated using lysis buffer (50 mM HEPES, $50 \mathrm{mM} \mathrm{NaCl}, 5 \mathrm{mM}$ EDTA, 1\% Triton $\mathrm{X}-100,1 \mathrm{mM} \mathrm{Na} \mathrm{VO}_{4}, 50 \mathrm{mM} \mathrm{NaF}, 10 \mathrm{mM}$ sodium 
B
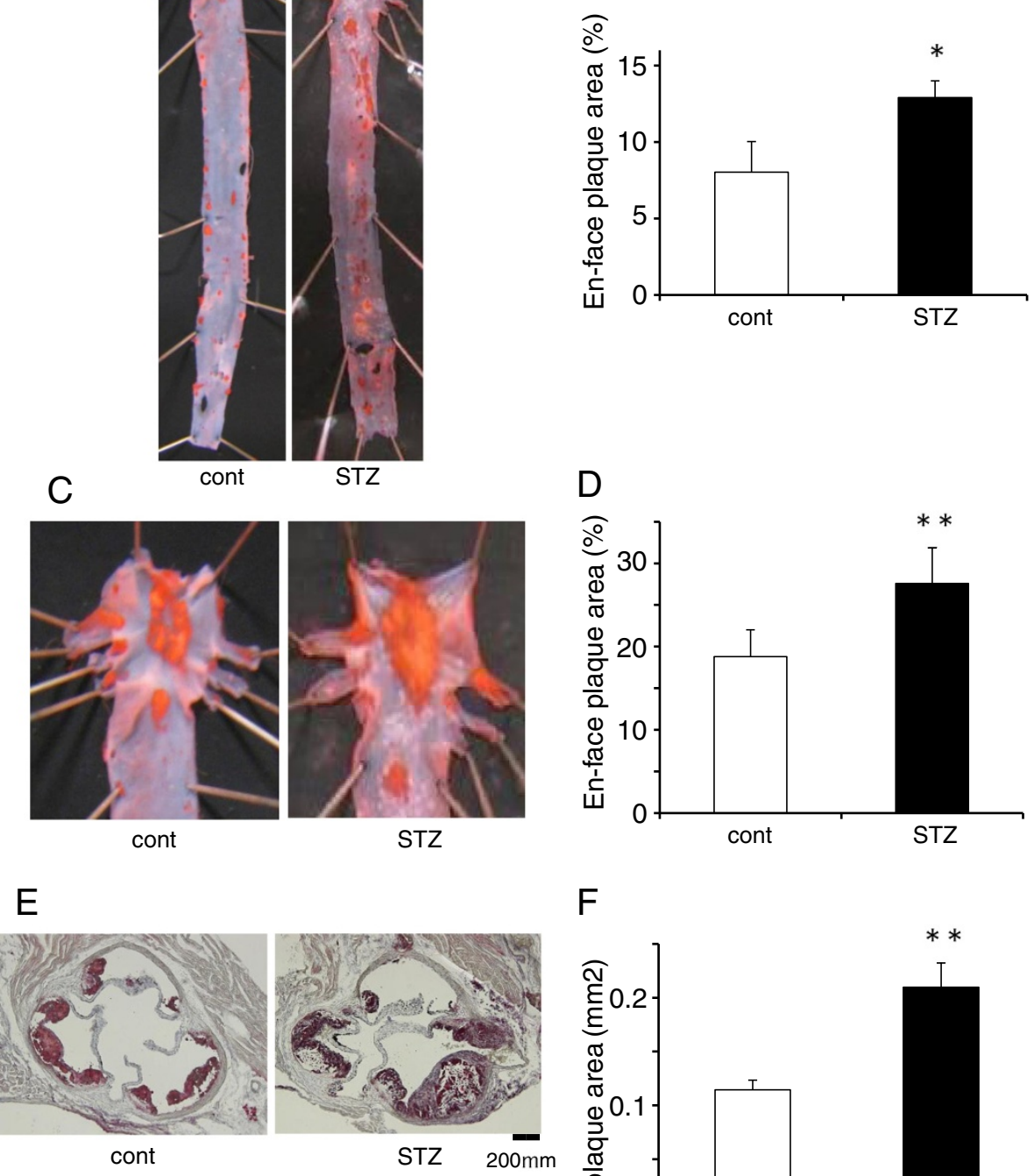

A

C

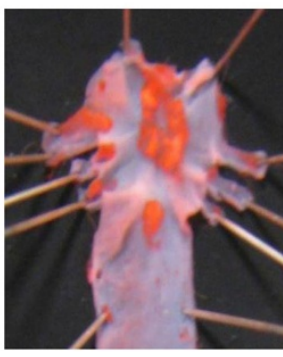

cont
$\mathrm{F}$

STZ $200 \mathrm{~mm}$

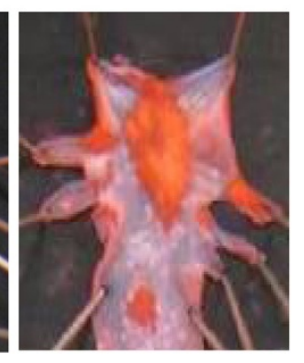

STZ

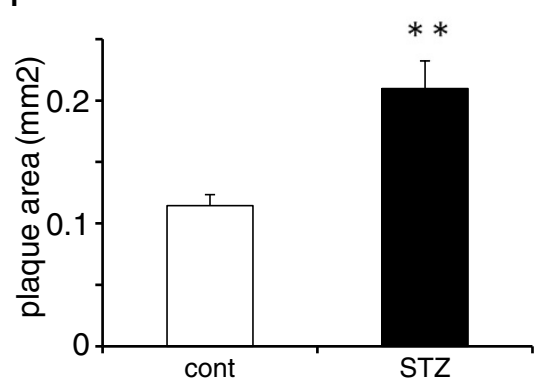

Figure 2 Atherosclerotic plaques in the aorta. (A, C, E) Representative photomicrographs of control (cont) and diabetic ApoE KO mice (STZ) showing that STZ-induced diabetes accelerates the progression of atherosclerotic lesions in the whole aorta, aortic arch, and aortic root. (B, D, F) Quantitative measurement of the en face plaque area (\%) in the whole aorta (B) and aortic arch (D), and oil red-O-stained plaque area ( $\left.\mathrm{mm}^{2}\right)$ in the aortic root (F) ( $n=9$ mice/group). Each bar indicates mean \pm S.D. ${ }^{*} P<0.05$ and ${ }^{* *} P<0.01$ vs control mice.

pyrophosphate decahydrate and $1 \mathrm{mM}$ phenylmethylsulfonyl fluoride), containing $1 \%$ phosphatase inhibitor cocktail 1 (Sigma), 1\% phosphatase inhibitor cocktail 2 (Sigma), and $1 \%$ protease inhibitor cocktail (Sigma). After tissue homogenization, particulate material was removed by centrifugation, and protein concentrations measured using the Bio-Rad protein assay (Bio-Rad Laboratories, Hercules, CA, USA). Equal amounts of total protein (30 $\mathrm{\mu g} / \mathrm{sample}$ ) were subjected to sodium dodecyl sulfate-polyacrylamide gel electrophoresis (10\%) and electrophoretically transferred to polyvinylidene difluoride membranes (Millipore, Billerica, MA, USA). Non-specific antibody binding was blocked by incubating the membranes with Blocking One (Nacalai Tesque) for $60 \mathrm{~min}$ at room temperature. The primary antibodies used were mouse monoclonal anti-BMP4 (1:100, Santa Cruz Biotechnology Inc.) and anti-SMAD1 (1:100, Santa Cruz Biotechnology Inc.), and rabbit polyclonal antiphospho-SMAD1/5/8 (1:1000, Cell Signaling, Danvers, MA) and anti- $\beta$-actin (1:2000, Abcam, Cambridge, UK). Blots were incubated overnight at $4{ }^{\circ} \mathrm{C}$ with primary antibodies, and then incubated with horseradish 


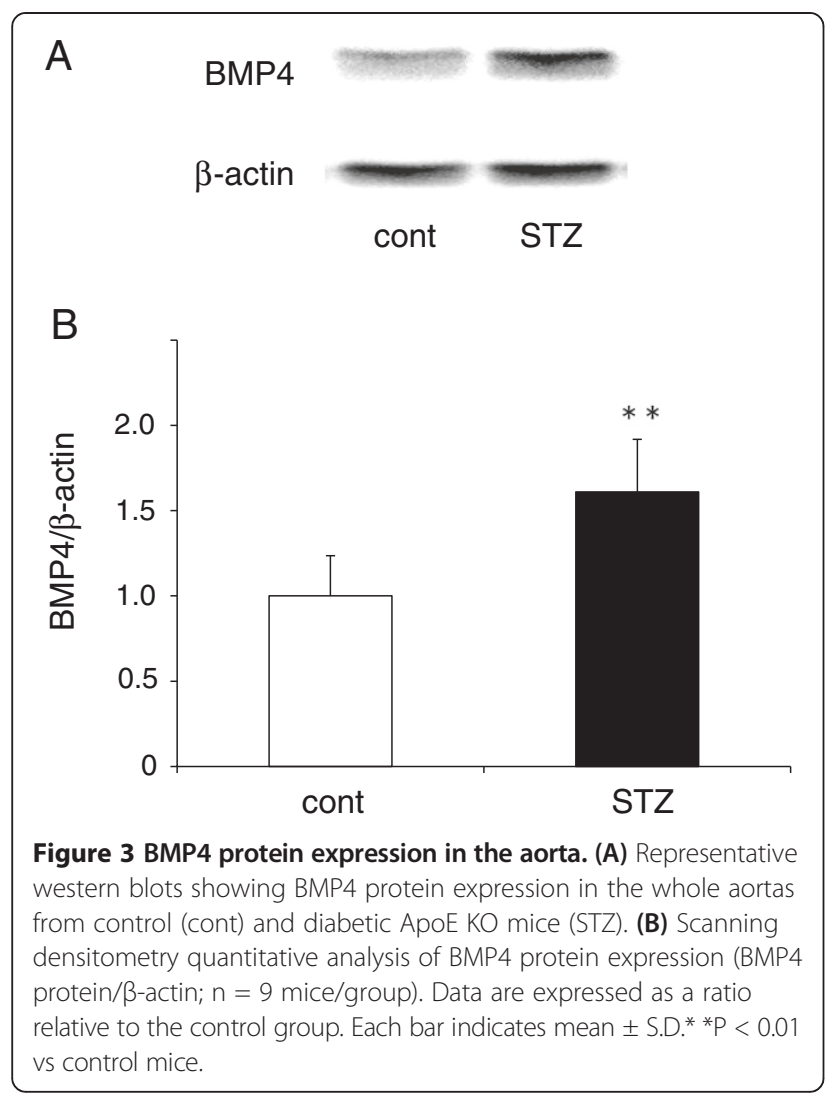

peroxidase-conjugated secondary antibodies (1:5000 dilution) for $60 \mathrm{~min}$ at room temperature. The blot was developed using an ECL detection kit (Amersham International, Little Chalfont, UK). Signal intensities were normalized using beta-actin. Band images were digitally captured with a FluorChem SP imaging system (Alpha Innotech, San Leandro, CA, USA) and band intensities quantified using Image $J$ software.

\section{Preparation of peritoneal macrophages}

To isolate peritoneal macrophages, we intraperitoneally injected wild-type mice with $2 \mathrm{~mL}$ of $4 \%$ thioglycollate. Cells were collected from the peritoneal cavity 3 days after injection and were incubated in 12-well plates in complete medium (RPMI1640 media containing 10\% fetal bovine serum and $100 \mathrm{U} / \mathrm{mL}$ penicillin/streptomycin). After $2 \mathrm{~h}$, the cells were washed three times with PBS and cultured in media. The adherent cells, considered to be peritoneal macrophages, were used in the experiments [26]. For a single experiment, peritoneal macrophages were collected from one mouse.

Uptake of oxLDL into peritoneal macrophages OxLDL was purchased from HARBOR BIO-PRODUCTS (Norwood, MA, USA). Recombinant BMP4 and Noggin (a BMP4 antagonist) were purchased from R\&D systems (Minneapolis, MN, USA). Peritoneal macrophages were serum starved for $3 \mathrm{~h}$ and then pretreated with or without $150 \mathrm{ng} / \mathrm{ml}$ Noggin for $3 \mathrm{~h}$. OxLDL $(15 \mu \mathrm{g} / \mathrm{ml})$ and BMP4 (100ng/ml) were added to Noggin-treated or untreated peritoneal macrophages for $20 \mathrm{~h}$. After washing three times with PBS, cells were fixed in $4 \%$ paraformaldehyde, and stained with Oil red-O and hematoxylin to evaluate uptake of oxLDL. The average percentage of Oil red O-labeled macrophages for each well was obtained by counting the number of labeled and unlabeled cells in 15 microscopic fields selected from each of three separate wells/group. The average value was calculated for each group. The experiment was repeated four times and the average percentage of four separate experiments was shown in Figure $1 \mathrm{C}$.

\section{Measurement of blood glucose and plasma cholesterol levels}

At 12 weeks of age, blood was collected to measure blood glucose, plasma total cholesterol and triglyceride levels. Glucose was measured directly from the tail tip with a glucometer (Glutest sensor, Sanwa Kagaku Kenkyusho, Mie, Japan). Plasma total cholesterol and triglyceride levels were determined using commercial available kits (Wako Chemical Co., Osaka, Japan).

\section{Statistical analysis}

All quantitative analyses were performed by a single observer blinded to the experimental protocol. Data are expressed as means \pm standard deviation. Differences among the two groups were compared using unpaired Student's $t$-test. The statistic comparison among the 8 groups was performed using ANOVA followed by Tukey's Multiple Comparison tests. Values of $\mathrm{P}<0.01$ were considered to be statistically significant.

\section{Results}

Plasma lipid and lipoprotein levels in diabetic ApoE KO mice

ApoE KO mice were rendered diabetic with an intraperitoneal administration of STZ, and were then fed a high-fat diet for 4 weeks. Approximately $80 \%$ of the mice were diabetic at 2 weeks after STZ administration, defined as non-fasting glucose $>250 \mathrm{mg} / \mathrm{dL}$. Six weeks after STZ, glucose levels in the control and diabetic ApoE KO mice were $102 \pm 12$ and $454 \pm 123 \mathrm{mg} / \mathrm{dL}$, respectively (Table 1). After being fed the high-fat diet for 4 weeks, fasting plasma cholesterol levels in the diabetic ApoE $\mathrm{KO}$ mice were 1.4-fold higher than those in the control mice $(2529 \pm 513$ vs. $1815 \pm 302 \mathrm{mg} / \mathrm{dL})$. Plasma triglyceride levels were not significantly different between the two groups of mice $(\mathrm{n}=18$, control mice; $\mathrm{n}=16$, diabetic ApoE KO mice). 


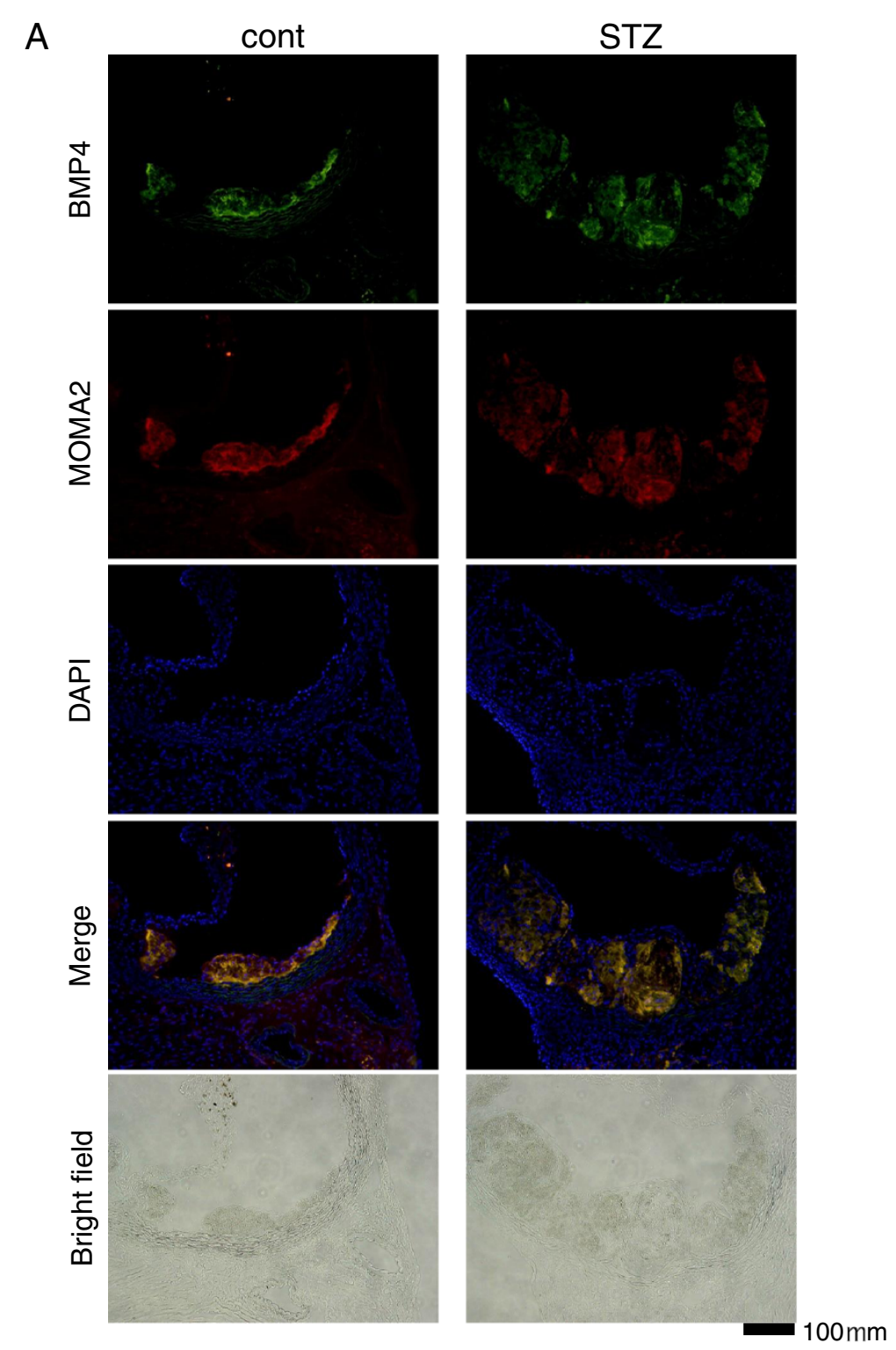

B

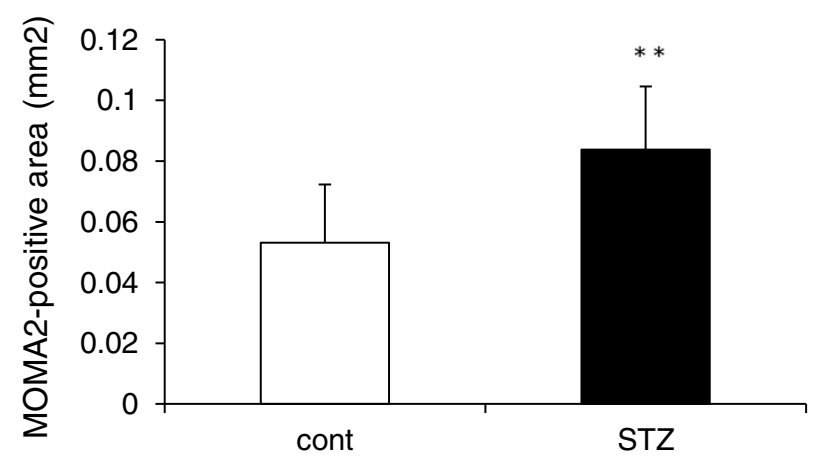

Figure 4 Expression of BMP4 in monocytes/macrophages in the aortic roots of control (cont) and diabetic ApoE KO mice (STZ). (A) Representative images showing the localization of BMP4 (green), MOMA2 (red) and DAPI (blue) staining in each group. Frozen sections of the aortic roots were stained with anti-BMP4 and anti-MOMA2 antibodies, and were then observed under a fluorescence microscope. (B) In each group, the MOMA2-positive area $\left(\mathrm{mm}^{2}\right)$ in the aortic root was measured in five separate sections from 8 mice. Results are presented as the average area. Each bar indicates mean \pm S.D. ${ }^{* *} \mathrm{P}<0.01$ vs control mice. 


\section{Atherosclerotic plaques in the aorta}

At 12 weeks of age, the control and diabetic ApoE $\mathrm{KO}$ mice were killed to measure plaque area. The non-diabetic control ApoE KO mice had Oil red O-stained atherosclerotic plaques extending from the ascending aorta to the aortic arch. By contrast, the diabetic mice had more severe aortic plaque formation, with an increase in en face plaque area of 15\% (Figure 2A-D). In addition, STZ-induced diabetes markedly accelerated the formation of atherosclerotic plaques in the aortic sinus (Figure 2E,F).

\section{BMP4 protein expression in the aorta}

BMP4 protein expression levels in the aortas of control and diabetic ApoE KO mice were evaluated by western blot. Aortic BMP4 protein expression was significantly increased, by approximately 60\%, in diabetic ApoE KO mice compared with control ApoE KO mice ( $\mathrm{n}=9$ mice/group) (Figure 3B). Cross-sections taken at the aortic sinus were evaluated to determine the structural features of the atherosclerotic lesions. At 12 weeks of age, the plaques in the aortic root consisted of a lipid-rich core and massive macrophage infiltration into the intima in control ApoE KO mice. By contrast, the formation of lipid- and macrophage-rich plaques was remarkably increased at the aortic root in the diabetic ApoE KO mice.

Next, to test whether BMP4 was expressed in atherosclerotic lesions in the aortic sinus of ApoE KO mice, we stained several sections of the aortic root with anti-BMP4 antibody. The low magnification images (Figure 4A) revealed BMP4 protein in the internal layer. The localization of BMP4 in the atherosclerotic lesion was confirmed by double-immunofluorescence staining with anti-MOMA2 antibody (specific for monocytes/macrophages) and anti-BMP4 antibody. The BMP4-positive area appeared to correspond to the macrophage-rich area of the atherosclerotic lesion in the aortic root (Figure 4A). Furthermore, MOMA2-stained areas were larger at the aortic root in diabetic ApoE $\mathrm{KO}$ mice, than in controls (Figure 4B). BMP4 expression in monocytes/macrophages in the atherosclerotic lesions was much greater in diabetic mice than in control ApoE $\mathrm{KO}$ mice.

\section{Phosphorylation of SMAD1/5/8 signaling in the aorta}

Western blot analysis showed SMAD1/5/8 phosphorylation was clearly induced in the whole aorta in diabetic ApoE KO mice, compared with controls (Figure 5A). Summarized data of SMAD1/5/8 phosphorylation is shown (Figure $5 \mathrm{~B} ; \mathrm{n}=6 /$ group).

\section{BMP4 increases oxLDL uptake in the peritoneal macrophages}

OxLDL incorporation in peritoneal macrophages obtained from wild-type mice was markedly increased by BMP4 treatment compared with untreated peritoneal
A

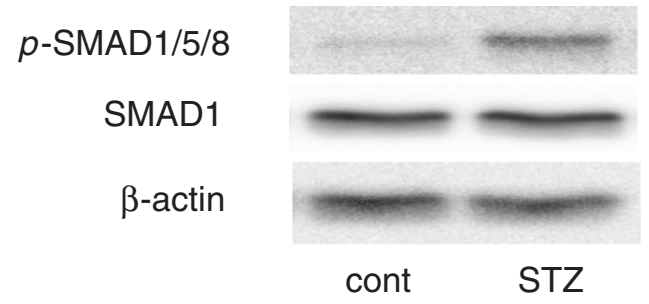

B

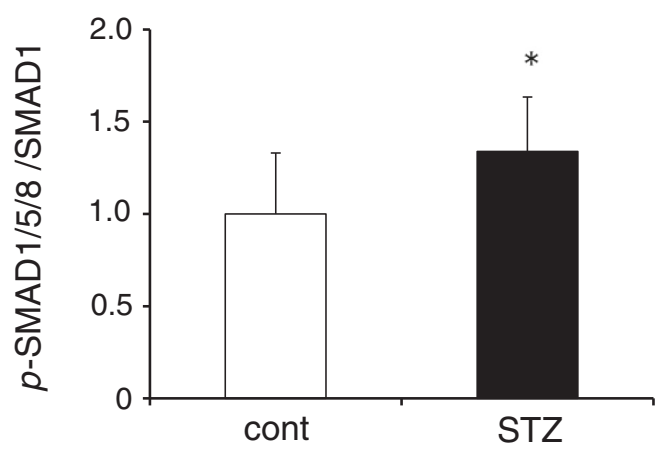

Figure 5 The BMP4 signaling pathway is activated in atherosclerotic lesions in STZ group. Representative western blots (A) and densitometric analysis (B) of p-SMAD1/5/8 and SMAD1 in each group ( $n=6$ mice/group). A ratio of $p-S M A D 1 / 5 / 8$ to SMAD1 was calculated from band intensities and data expressed as a ratio relative to the control group ( $n=6$ mice/group). Each bar indicates mean \pm S.D. ${ }^{*} P<0.05$ vs control mice.

macrophages (Figure 1). Noggin, a BMP4 antagonist, inhibited BMP4-induced oxLDL uptake in peritoneal macrophages. In the absence of oxLDL, few Oil red$\mathrm{O}$-positive peritoneal macrophages were observed in each group. On the other hand, we observed few Oil red-O-positive peritoneal macrophages in the absence of oxLDL. BMP4 alone did not increase the number of Oil red-O positive peritoneal macrophages (Figure 1).

\section{Discussion}

Diabetes leads to the progression of atherosclerotic lesions, coronary artery disease, stroke, and peripheral vascular disease [27-29]. Atherosclerosis, an inflammatory disease, is thought to occur as a result of the uptake of oxLDL into macrophages/monocytes [30-33]. Current clinical strategies have focused on lipid lowering with statins, for example, to prevent the progression of atherosclerosis.

The present study provided the first experimental evidence to show that BMP4 enhances oxLDL uptake into peritoneal macrophages. We also found that BMP4 protein expression was markedly upregulated in the aorta of STZ-induced diabetic ApoE KO mice, compared with controls (Figure 3). Recent findings suggest that BMP4 may function as a pro-inflammatory and pro-atherogenic 
vasculature mediator [15]. We showed that BMP4 protein expression was elevated (Figure 4A) in parallel with increased accumulation of MOMA2-stained macrophages (Figure 4B) in atherosclerotic plaques from diabetic ApoE $\mathrm{KO}$ mice. These findings suggest that increased BMP4 expression in aortic macrophages of diabetic ApoE $\mathrm{KO}$ mice, may be involved in enhanced oxLDL uptake. In the present study, we induced diabetes in ApoE $\mathrm{KO}$ atherosclerotic mice by injecting them with STZ $[22,23]$. These mice developed marked hyperglycemia, with blood glucose levels $>250 \mathrm{mg} / \mathrm{dL}$. STZ also increased the plasma total cholesterol levels in the ApoE KO mice but did not affect triglyceride levels compared with the control ApoE KO mice (Table 1).

As shown in Figure 2, atherosclerotic plaque formation was accelerated in the whole aorta, aortic arch, and aortic root of diabetic ApoE KO mice. These observations indicate that diabetes accelerates atherosclerotic plaque formation. BMP4 expression was also much greater in the whole aortas of diabetic ApoE $\mathrm{KO}$ mice compared with control mice (Figure 3), suggesting that diabetes also induces aortic BMP4 expression in $d b / d b$ mice [15]. BMP4 induces the activation of the SMAD1/5/8 signaling pathway. In this study, diabetic ApoE $\mathrm{KO}$ mice showed strong activation of BMP4/SMAD1/5/8 signaling in aortas compared with control ApoE $\mathrm{KO}$ mice due to increased expression of BMP4 in the diabetic aortas (Figures 3, 4, and 5). These data suggest that BMP4 may be one of the important regulators to progress plaque formation underlying diabetes diseases. There is evidence indicating that BMP antagonists and signaling pathway inhibitors block activation of SMAD1/5/8 signaling, and thereby reduce the incidence of subsequent events, including vascular inflammation and atherosclerosis [34,35]. These findings suggest that BMP signals are novel therapeutic targets for vascular inflammation and/or atherosclerosis.

To examine the localization of BMP4 expression in the aorta, we performed double-fluorescence staining of monocytes/macrophages and BMP4. The BMP4and monocyte/macrophage-positive areas were largely colocalized in the atherosclerotic plaque of aortic roots, as shown in Figure 4A. Lesional monocytes and macrophages are the main cell types involved in the progression of atherosclerotic plaques, because the phagocytic activity of macrophages in the plaque contributes to the development of atherosclerosis and plaque instability. BMP4 treatment increased 2.6-fold the number of cells with oxLDL uptake, when compared with controls (12\% of the total) (Figure 1). This marked increase in macrophages showing oxLDL uptake was significantly inhibited by $50 \%$ when cells were treated with Noggin. These results suggest that the increase in BMP4 expression associated with diabetes will enhance the uptake of oxLDL into macrophages in atherosclerotic lesions.
Therefore, it is very likely that diabetes accelerates the formation of atherosclerotic plaques and lowers the threshold for destabilization and rupture of atherosclerotic lesions.

In conclusion, we have demonstrated that BMP4 is expressed in monocytes/macrophages in atherosclerotic plaques in a mouse model of diabetes and atherosclerosis. We also found that BMP4 enhances oxLDL uptake into peritoneal macrophages in vitro. The induction of BMP4 in atherosclerotic plaque may promote atherosclerotic plaque formation in diabetes. These findings raise the possibility that inhibition of BMP4 signaling may represent a potential therapeutic target for atherosclerosis and other diseases associated with BMPs and diabetes.

\section{Competing interests}

The authors declare that they have no competing interests.

\section{Authors' contributions}

MK: Conceived, designed and conducted experiments for the study and wrote the manuscript. YK, RJ, AT, NT: Performed experiments and helped in constructing the figures. TN: Participated in the study design and coordination and drafting of manuscript. AY: Contributed to supervision of laboratory procedures, data analysis and interpretation. YK: Conceived and designed the study, contributed to data analysis and interpretation and wrote the manuscript. All authors have read and approved this manuscript.

\section{Acknowledgment}

The authors thank Ms. Yuma Ohkido and Ms. Naoka Kubo for technical assistance.

Grants: This work was supported in part by Grants-in-Aid for Scientific Research [(to YK) (C) 22590255], and Funds [(to MK) no.111502] from the central research institute of Fukuoka University.

Received: 27 December 2012 Accepted: 4 October 2013

Published: 9 October 2013

\section{References}

1. Haffner SM, Lehto S, Ronnemaa T, Pyorala K, Laakso M: Mortality from coronary heart disease in subjects with type 2 diabetes and in nondiabetic subjects with and without prior myocardial infarction. N Engl J Med 1998, 339:229-234.

2. Mitra S, Goyal T, Mehta JL: Oxidized LDL, LOX-1 and atherosclerosis. Cardiovasc Drugs Ther 2011, 25:419-429.

3. Yla-Herttuala S: Macrophages and oxidized low density lipoproteins in the pathogenesis of atherosclerosis. Ann Med 1991, 23:561-567.

4. Jialal I, Devaraj S: The role of oxidized low density lipoprotein in atherogenesis. J Nutr 1996, 126:1053S-1057S.

5. Frostegard J, Nilsson J, Haegerstrand A, Hamsten A, Wigzell H, Gidlund M: Oxidized low density lipoprotein induces differentiation and adhesion of human monocytes and the monocytic cell line U937. Proc Natl Acad Sci U S A 1990, 87:904-908.

6. Frostegard J, Wu R, Giscombe R, Holm G, Lefvert AK, Nilsson J: Induction of T-cell activation by oxidized low density lipoprotein. Arterioscler Thromb 1992, 12:461-467.

7. Chen D, Zhao M, Mundy GR: Bone morphogenetic proteins. Growth Factors 2004, 22:233-241.

8. Massague J, Chen YG: Controlling TGF-beta signaling. Genes Dev 2000, 14:627-644.

9. Ebendal $\mathrm{T}$, Bengtsson $\mathrm{H}$, Soderstrom $\mathrm{S}$ : Bone morphogenetic proteins and their receptors: potential functions in the brain. J Neurosci Res 1998, 51:139-146.

10. Graff JM: Embryonic patterning: to BMP or not to BMP, that is the question. Cell 1997, 89:171-174.

11. Hogan BL: Bone morphogenetic proteins: multifunctional regulators of vertebrate development. Genes Dev 1996, 10:1580-1594. 
12. Kiyono M, Shibuya M: Bone morphogenetic protein 4 mediates apoptosis of capillary endothelial cells during rat pupillary membrane regression. Mol Cell Biol 2003, 23:4627-4636.

13. Wozney JM, Rosen $\mathrm{V}$ : Bone morphogenetic protein and bone morphogenetic protein gene family in bone formation and repair. Clin Orthop Relat Res 1998, 346:26-37.

14. Derwall M, Malhotra R, Lai CS, Beppu Y, Aikawa E, Seehra JS, Zapol WM, Bloch KD, Yu PB: Inhibition of bone morphogenetic protein signaling reduces vascular calcification and atherosclerosis. Arterioscler Thromb Vasc Biol 2012, 32:613-622.

15. San Martin A, Du P, Dikalova A, Lassegue B, Aleman M, Gongora MC, Brown K, Joseph G, Harrison DG, Taylor WR, et al: Reactive oxygen speciesselective regulation of aortic inflammatory gene expression in Type 2 diabetes. Am J Physiol Heart Circ Physiol 2007, 292:H2073-H2082.

16. Shao JS, Cai J, Towler DA: Molecular mechanisms of vascular calcification: lessons learned from the aorta. Arterioscler Thromb Vasc Biol 2006, 26:1423-1430.

17. Sorescu GP, Sykes M, Weiss D, Platt MO, Saha A, Hwang J, Boyd N, Boo YC, Vega JD, Taylor WR, Jo H: Bone morphogenic protein 4 produced in endothelial cells by oscillatory shear stress stimulates an inflammatory response. J Biol Chem 2003, 278:31128-31135.

18. Dhore CR, Cleutjens JP, Lutgens E, Cleutjens KB, Geusens PP, Kitslaar PJ, Tordoir JH, Spronk HM, Vermeer C, Daemen MJ: Differential expression of bone matrix regulatory proteins in human atherosclerotic plaques. Arterioscler Thromb Vasc Biol 2001, 21:1998-2003.

19. Vendrov AE, Madamanchi NR, Hakim ZS, Rojas M, Runge MS: Thrombin and $\mathrm{NAD}(\mathrm{P}) \mathrm{H}$ oxidase-mediated regulation of CD44 and BMP4-Id pathway in VSMC, restenosis, and atherosclerosis. Circ Res 2006, 98:1254-1263.

20. Miriyala S, Gongora Nieto MC, Mingone C, Smith D, Dikalov S, Harrison DG, Jo H: Bone morphogenic protein-4 induces hypertension in mice: role of noggin, vascular NADPH oxidases, and impaired vasorelaxation. Circulation 2006, 113:2818-2825.

21. Chang K, Weiss D, Suo J, Vega JD, Giddens D, Taylor WR, Jo H: Bone morphogenic protein antagonists are coexpressed with bone morphogenic protein 4 in endothelial cells exposed to unstable flow in vitro in mouse aortas and in human coronary arteries: role of bone morphogenic protein antagonists in inflammation and atherosclerosis. Circulation 2007, 116:1258-1266.

22. Ding H, Hashem M, Wiehler WB, Lau W, Martin J, Reid J, Triggle C: Endothelial dysfunction in the streptozotocin-induced diabetic apoE-deficient mouse. Br J Pharmacol 2005, 146:1110-1118.

23. Goldberg IJ, Hu Y, Noh HL, Wei J, Huggins LA, Rackmill MG, Hamai H, Reid BN, Blaner WS, Huang LS: Decreased lipoprotein clearance is responsible for increased cholesterol in LDL receptor knockout mice with streptozotocin-induced diabetes. Diabetes 2008, 57:1674-1682.

24. Koga M, Kai H, Yasukawa H, Yamamoto T, Kawai Y, Kato S, Kusaba K, Kai M, Egashira K, Kataoka Y, Imaizumi T: Inhibition of progression and stabilization of plaques by postnatal interferon-gamma function blocking in ApoE-knockout mice. Circ Res 2007, 101:348-356.

25. Koga M, Kai H, Yasukawa H, Kato S, Yamamoto T, Kawai Y, Kusaba K, Seki Y, Kai M, Egashira K, et al: Postnatal blocking of interferon-gamma function prevented atherosclerotic plaque formation in apolipoprotein Eknockout mice. Hypertens Res 2007, 30:259-267.

26. Shaw DR, Griffin FM Jr: Thioglycollate-elicited mouse peritoneal macrophages are less efficient than resident macrophages in antibodydependent cell-mediated cytolysis. J Immunol 1982, 128:433-440.

27. Slavina ES, Madanat A, Pankov Yu A, Syrkin AL, Tertov W, Orekhov AN: Diabetes mellitus and atherosclerosis. N Engl J Med 1987, 317:836.

28. Hansson GK: Inflammation, atherosclerosis, and coronary artery disease. N Engl J Med 2005, 352:1685-1695.

29. ladecola C, Anrather J: The immunology of stroke: from mechanisms to translation. Nat Med 2011, 17:796-808.

30. Ross R: Atherosclerosis is an inflammatory disease. Am Heart J 1999, 138:S419-S420.

31. Ross R: Atherosclerosis-an inflammatory disease. N Engl J Med 1999, 340:115-126.

32. Libby P: Inflammation in atherosclerosis. Nature 2002, 420:868-874

33. Tahara N, Kai H, Ishibashi M, Nakaura H, Kaida H, Baba K, Hayabuchi N, Imaizumi T: Simvastatin attenuates plaque inflammation: evaluation by fluorodeoxyglucose positron emission tomography. J Am Coll Cardiol 2006, 48:1825-1831.
34. Bostrom Kl, Jumabay M, Matveyenko A, Nicholas SB, Yao Y: Activation of vascular bone morphogenetic protein signaling in diabetes mellitus. Circ Res 2011, 108:446-457

35. Yao Y, Shao ES, Jumabay M, Shahbazian A, Ji S, Bostrom Kl: High-density lipoproteins affect endothelial BMP-signaling by modulating expression of the activin-like kinase receptor 1 and 2. Arterioscler Thromb Vasc Biol 2008, 28:2266-2274

doi:10.1186/1476-9255-10-32

Cite this article as: Koga et al:: BMP4 is increased in the aortas of diabetic ApoE knockout mice and enhances uptake of oxidized low density lipoprotein into peritoneal macrophages. Journal of Inflammation 2013 10:32.

\section{Submit your next manuscript to BioMed Central and take full advantage of:}

- Convenient online submission

- Thorough peer review

- No space constraints or color figure charges

- Immediate publication on acceptance

- Inclusion in PubMed, CAS, Scopus and Google Scholar

- Research which is freely available for redistribution

Submit your manuscript at www.biomedcentral.com/submit
C) Biomed Central 